\title{
DPP-4 Inhibitor-associated Bullous Pemphigoid in a Hemodialysis Patient
}

\author{
Jun Usami ${ }^{1}$ and Yumiko Takezawa ${ }^{2}$ \\ Key words: dipeptidyl peptidase (DPP)-4 inhibitor, vildagliptin, bullous pemphigoid, hemodialysis
}

(Intern Med 59: 593, 2020)

(DOI: 10.2169/internalmedicine.3461-19)
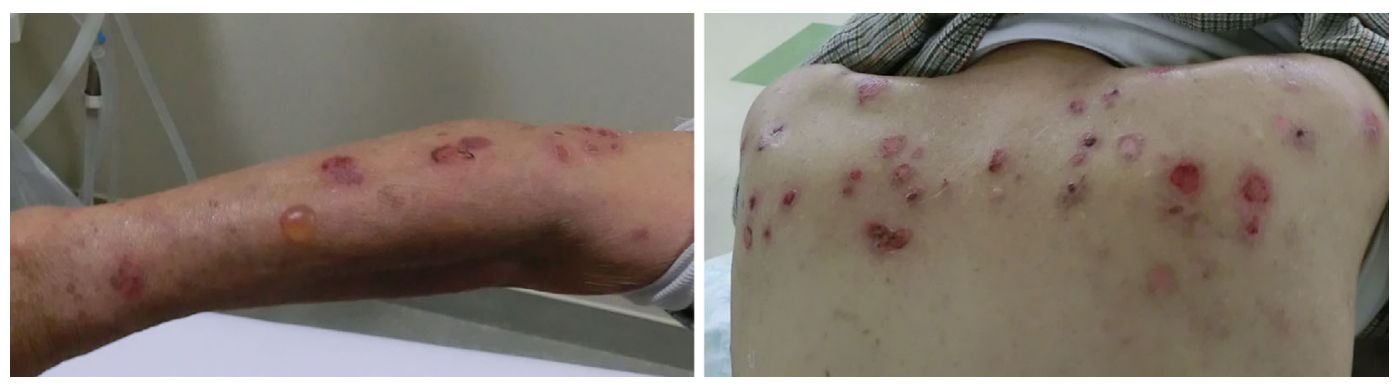

Picture.

An 85-year-old man complained of a rash on his upper limbs, chest wall and back (Picture). He had been undergoing hemodialysis due to end-stage diabetic kidney disease and taking vildagliptin [dipeptidyl peptidase-4 inhibitor (DPP4i)] for more than a year. DPP4is are incretin-related drugs widely prescribed for the treatment of type 2 diabetes. In time, his rash gradually spread over the rest of his body. Although a skin biopsy was not performed, a dermatologist confidently diagnosed him with non-inflammatory bullous pemphigoid (BP), which included tense blisters and a small number of erythemas. The patient's anti-BP180 NC16a antibody serum level was negative $[<3.0 \mathrm{U} / \mathrm{mL}$, chemiluminescence enzyme immuno assay (CLEIA) method]. BP is an autoimmune subepidermal disease typically characterized by inflamed, itchy edematous erythema and tense blisters. Some drugs [e.g., diuretics and DPP4is (1)] may be potential triggers. No other drugs that could possibly cause BP, except for the DPP4i, had been prescribed to him. The relationship between BP and hemodialysis itself is unclear at present. Recently, it was found that patients with DPP4iassociated BP (DPP4i-BP) tend to exhibit a noninflammatory phenotype that presents with fewer erythemas. In addition, the anti-BP180 NC16a antibody serum levels are often low or even negative in patients with DPP4i-BP, although they are usually positive in patients with typical BP (2). We therefore concluded that the patient's rash had been caused by the vildagliptin, so its administration was immediately stopped, and the daily oral administration of 20 $\mathrm{mg}$ of prednisolone was started. The patient's symptoms gradually resolved. There are few case reports of BP caused by vildagliptin administration in dialysis patients.

Written consent for publication was obtained from the patient.

The authors state that they have no Conflict of Interest (COI).

\section{References}

1. Béné J, Moulis G, Bennani I, et al. Bullous pemphigoid and dipeptidyl peptidase IV inhibitors: a case-noncase study in the French Pharmacovigilance Database. Br J Dermatol 175: 296-301, 2016.

2. Horikawa H, Kurihara Y, Funakoshi T, et al. Unique clinical and serological features of bullous pemphigoid associated with dipeptidyl peptidase-4 inhibitors. Br J Dermatol 178: 1462-1463, 2018.

The Internal Medicine is an Open Access journal distributed under the Creative Commons Attribution-NonCommercial-NoDerivatives 4.0 International License. To view the details of this license, please visit (https://creativecommons.org/licenses/ by-nc-nd/4.0/).

${ }^{1}$ General Medicine, Aichi Medical University, Japan and ${ }^{2}$ Takezawa Clinic, Japan

Received: June 12, 2019; Accepted: August 27, 2019; Advance Publication by J-STAGE: October 15, 2019

Correspondence to Dr. Jun Usami, jusami176@ hotmail.com

(C) 2020 The Japanese Society of Internal Medicine. Intern Med 59: 593, 2020 